\title{
Satisfacción laboral del docente universitario desde la cultura organizacional
}

\author{
Job satisfaction of the university teacher from the organizational
}

Satisfação no trabalho do professor universitário a partir da cultura organizacional

Angélica Díaz Tinoco ${ }^{1}$

adiazt@unac.edu.pe

https://orcid.org/0000-0001-9915-3418

Olga Giovanna Valderrama Rios ${ }^{1}$

ogvalderramar@unac.edu.pe

https://orcid.org/0000-0003-1906-7101

\section{Alicia Lourdes Merino Lozano ${ }^{1}$ \\ amerinope@gmail.com \\ https://orcid.org/0000-0003-4430-0104}

\section{Llerme Nuñez Zarazú}

opebri@yahoo.com

https://orcid.org/0000-0002-8011-5072

\author{
Juana Matilde Cuba Sancho ${ }^{3}$ \\ jcubas@unmsm.edu.pe \\ https://orcid.org/0000-0002-9803-1571 \\ ${ }^{1}$ Universidad Nacional del Callao, Lima- Perú \\ ${ }^{2}$ Universidad Nacional Santiago Antúnez de Mayolo, Ancash-Perú \\ ${ }^{3}$ Universidad Nacional Mayor de San Marcos, Lima - Perú
}

Artículo recibido 2 de noviembre 2021, arbitrado y aceptado 24 de noviembre 2021 y publicado 7 de marzo 2022

\section{RESUMEN}

Elpropósito de estudio esidentificarla relación de la cultura organizacional en la satisfacción laboral de los docentes de una universidad de la costa peruana. Investigación descriptiva, correlacional y transversal. La muestra fue de 48 docentes, obtenidos mediante muestreo aleatorio simple. Los instrumentos utilizados fueron dos; la escala de satisfacción laboral SL-SPC y otro para cultura organizacional, ambos en escala de Likert. Para los análisis estadísticos se usó la correlación de Spearman. Como resultado se obtuvo que el $66.7 \%$ presentó una cultura organizacional neutra, el $20.8 \%$ fuerte y el $12.5 \%$ débil. El 79.2\% indicaron tener una satisfacción laboral media, $14.6 \%$ alta y solo el $6.3 \%$ baja. La cultura organizacional se relacionó con la satisfacción laboral de los docentes $(r=0,375$, $p=0,009$ ). Se concluye Existe una correlación entre la cultura organizacional la satisfacción laboral, actividades en la primera mejoraran la segunda.

Palabras clave: Cultura Organizacional; Satisfacción laboral; Docentes; Universidad

\section{ABSTRACT}

The purpose of this study is to identify the relationship between organizational culture and job satisfaction among teachers at a university on the Peruvian coast. This was a descriptive, correlational and crosssectional research. The sample consisted of 48 teachers, obtained by simple random sampling. Two instruments were used: the SL-SPC job satisfaction scale and another for organizational culture, both on a Likert scale. Spearman's correlation was used for statistical analysis. The results showed that $66.7 \%$ had a neutral organizational culture, $20.8 \%$ had a strong one and $12.5 \%$ had a weak one. A total of $79.2 \%$ indicated medium job satisfaction, $14.6 \%$ high and only $6.3 \%$ low. Organizational culture was related to teachers' job satisfaction $(\mathrm{r}=0.375, \mathrm{p}=0.009)$. We conclude that there is a correlation between organizational culture and job satisfaction; activities in the former will improve the latter.

Key words: Organizational culture; Job satisfaction; Teachers; University

\section{RESUMO}

O objetivo deste estudo é identificar a relação entre cultura organizacional e satisfação profissional entre os professores de uma universidade na costa peruana. Esta é uma pesquisa descritiva, correlacional e transversal. A amostra consistiu de 48 professores, obtidos por simples amostragem aleatória. Dois instrumentos foram utilizados: a escala de satisfação profissional SL-SPC e outro para a cultura organizacional, ambos na escala Likert. A correlação do Spearman foi usada para análise estatística. Como resultado, 66,7\% tinham uma cultura organizacional neutra, $20,8 \%$ tinham uma cultura organizacional forte e $12,5 \%$ tinham uma cultura organizacional fraca. $79,2 \%$ indicaram satisfação profissional média, $14,6 \%$ de alta e apenas $6,3 \%$ de baixa. A cultura organizacional estava relacionada à satisfação do trabalho dos professores $(\mathrm{r}=0,375, \mathrm{p}=0,009)$. Conclui-se que existe uma correlação entre a cultura organizacional e a satisfação no trabalho, as atividades na primeira irão melhorar a segunda.

Palavras-chave: Cultura Organizacional; Satisfação no Trabalho; Professores; Universidade 


\section{INTRODUCCIÓN}

El estudio de la cultura organizacional es de gran valor fundamentalmente porque permite apreciar el trabajo a los docentes, generando un impacto en la motivación, satisfacción y producción del docente de la educación superior, por lo tanto, son las autoridades quienes deben realizar programas de desarrollo para que los docentes puedan potenciar sus competencias profesionales de ese modo puedan fortalecer sus valores y aumentando la retención de los buenos maestros. Una buena organización cuya base esté en valores y normas permite a cada uno de los miembros identificarse con la institución manteniendo conductas positivas e incrementando su productividad dando una buena imagen del lugar donde laboran, es decir, se logra un exitoso sistema de gestión.

La cultura organizacional a nivel académico implica la provisión de un servicio más que un producto. Los clientes de las organizaciones académicas se centran en los estudiantes donde es demostrado que la cultura organizacional tiene un impacto no solo en la investigación, sino incluso en la adopción de métodos de enseñanza innovadores.

Las universidades como centro principal y clave para la educación de los seres humanos, tienen una cultura especial como las demás organizaciones que puedan jugar un papel significativo en la formación de personas cualificadas, expertas y emprendedoras, por lo que requiere la existencia de una cultura organizacional adecuada en las Instituciones de Educación Superior. Estos centros preparan a los jóvenes para realizar cambios constantes en la sociedad y en el mundo, por lo que, la universidad tiene el enorme compromiso de responsabilidad cultural sobre su carga como institución cultural y creadora de cultura.
Pedraza, Obispo, Vásquez y Gómez (2015) definen la cultura organizacional se define como los supuestos básicos sobre el mundo y los valores que guían la vida en las organizaciones. Actualmente, las organizaciones afrontan diferentes fenómenos tal es el caso de la globalización la que tiene como consecuencia una alta complejidad del contexto global produciendo un impacto en los procesos sociales afectando el desarrollo de las personas en el ámbito laboral y por ende en la satisfacción laboral.

Las Instituciones de Educación Superior enfrentan grandes retos e induce grandes efectos sobre la capacidad para satisfacer con su función social lo que demanda garantizar atención especial a los diferentes procesos que desarrollan y a su gestión (Pedraza et al., 2015), lo cual demanda a la vez elevar la calidad de la planificación, la implementación y el control de la estrategia institucional mencionan Rodríguez, De León y Galarza (2015).

Desde el punto de vista de la Psicología (Pedraza et al., 2015), las declaraciones subjetivas de bienestar, en general, y de satisfacción laboral, en particular, han mostrado sus bondades como herramientas para el análisis del bienestar subjetivo de las personas (Nosnik, 2005). En este contexto se observó que los docentes de la Facultad de Ciencias de la Salud de la Universidad Nacional del Callao desde el punto de vista de la cultura organizacional, hay un desconocimiento de los principios y valores de la Universidad, se presentan inconformismos por ciertos manejos laborales, que se realizan en la organización en cuanto al desarrollo del talento humano o que puede generar dificultades en el cumplimiento de su misión y visión.

La satisfacción laboral de los profesores universitarios está relacionada con una serie de 
variables de función compleja, como los caracteres demográficos, el trabajo en sí mismo, el salario, las responsabilidades laborales, la variedad de tareas, las oportunidades de ratificación y promoción, la relación con los compañeros de trabajo y otros. Los profesores pueden estar simultáneamente satisfechos con algunas facetas del trabajo e insatisfechos con otros.

Es importante conocer cómo perciben los docentes el desarrollo de la propia cultura organizacional necesaria para cumplir con la misión y visión institucional para así elevar el nivel de la calidad educativa propiciando un clima de trabajo confortable, por lo que es necesario un estudio de investigación que permita interpretarla desde el punto de vista de sus miembros, como un primer acercamiento para que la organización educativa reflexione sobre sus futuras decisiones, acertadas o no, tomando en consideración las razones que inducen a su personal para pensar de esa manera y tomar una postura frente a su ambiente laboral.

En este contexto, la presente investigación determina la relación existente entre la relación entre la cultura organizacional y la satisfacción laboral de los docentes de una universidad pública de la costa peruana.

\section{MÉTODO}

Se realizó un estudio de enfoque cuantitativo, descriptivo, correlacional y transversal. La muestra fue de 48 profesores de la Facultad de Ciencias de la Salud de la Universidad Nacional del Callao de una población de 60, quienes aceptaron participar de manera voluntaria en el estudio. La técnica utilizada para la recolección de datos fue la encuesta, donde el instrumento para satisfacción laboral fue elaborado por Palma (2005), esta escala utiliza la técnica de
Likert, consta de 27 ítems. Para la variable cultural organizacional, se usó el instrumento elaborado por Olmos (2006). Fueron modificadas y validadas mediante un juicio de expertos, así como la confiabilidad fue evaluada mediante la prueba de Alfa de CronBach, en la que se obtuvo un valor de 0,80 , lo que indica que ambos instrumentos son fiables para la obtención de información y su alta consistencia interna. La modalidad de envío del cuestionario fue virtual relacionado al contexto de la pandemia en la que estamos vivenciando, y la recolección de datos fue durante el mes de setiembre del 2020. Se solicitaron los permisos a las autoridades de la Facultad y al comité de ética institucional.

Para el análisis y procesamiento de datos se utilizó el software estadístico SPSS 26.0 y se hizo uso de la estadística descriptiva e inferencial. Para determinar la asociación de las variables se tuvo en cuenta la correlación de Spearman. Asimismo, se respetó el derecho al anonimato y confidencialidad de los datos de los participantes.

\section{RESULTADOS}

La mayoría de los docentes $41.7 \%$ tiene una edad comprendida entre 51 y 60 años, el $75.0 \%$ son de sexo femenino, el $77.1 \%$ es casado, el $45.8 \%$ es docente a tiempo parcial y trabaja 20 horas mientras que el $43.8 \%$ de docentes tiene 21 años y más de tiempo de servicio como se muestra en el Gráfico 1.

En el Gráfico 2, con respecto a la cultura organizacional de los docentes de la Universidad Nacional del Callao, el $67 \%$ de los docentes evidencia una cultura organizacional neutra, el 21\% tienen una cultura organizacional fuerte, mientras que el $13 \%$ evidencian una cultura organizacional débil. 
De los 48 docentes encuestados, el 79\% satisfacción laboral alta y solo el 6\% docentes indicaron tener una satisfacción laboral media, presentaron una satisfacción laboral baja tal como seguido del $15 \%$ docentes presentaron una aparece reflejado en el Gráfico 3.

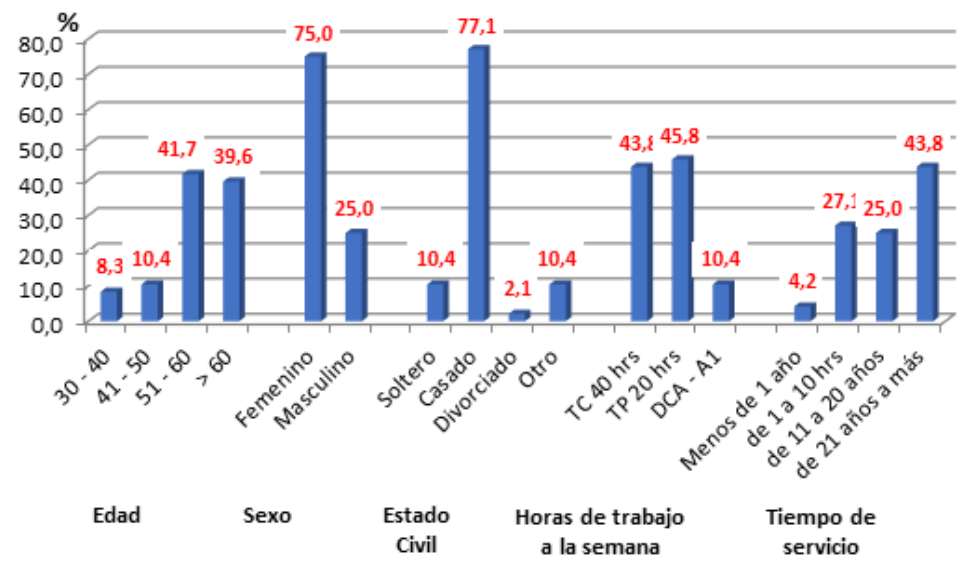

Gráfico 1. Características generales de los docentes de una universidad de la costa peruana.

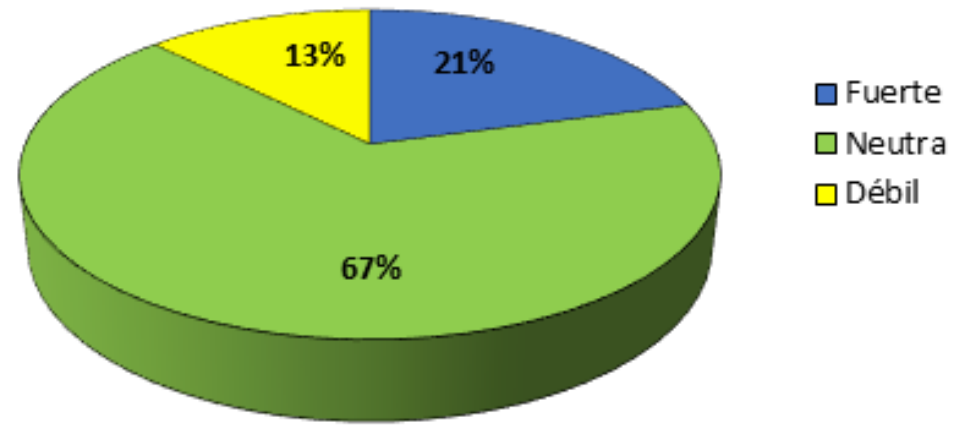

Gráfico 2. Cultura organizacional de los docentes de una universidad de la costa peruana.

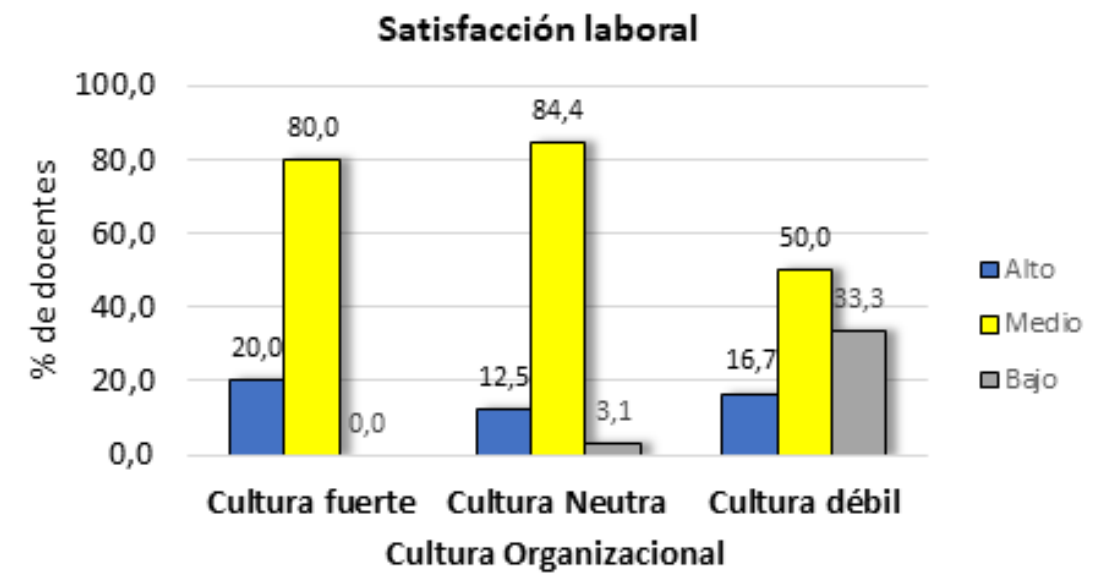

Gráfico 3. Porcentaje de docentes de una universidad de la costa peruana por satisfacción laboral, según cultura organizacional. 
En el Gráfico 4, se aprecia que del total de docentes que presentaron una cultura organizacional fuerte, el $80 \%$ presentó un nivel de satisfacción medio, el $20 \%$ presentó un nivel de satisfacción alto y ninguno presentó un nivel de satisfacción bajo, del total de docentes que presentó una cultura organizacional neutra el $84.4 \%$ presentó un nivel de satisfacción laboral medio, el $12.5 \%$ presentó un nivel de satisfacción alto y solo el 3.1\% presentó un nivel de satisfacción bajo. Del total de docentes que presentó una cultura organizacional débil, el $50.0 \%$ presentó un nivel de satisfacción medio, el $33.3 \%$ presentó un nivel de satisfacción bajo y solo el $16.7 \%$ presentó un nivel de satisfacción alto Esto con un análisis correlacional estadístico, determinó que la cultura organizacional se relacionó con la satisfacción laboral de los docentes, obteniendo un valor de 0,375 para un p de 0,009.

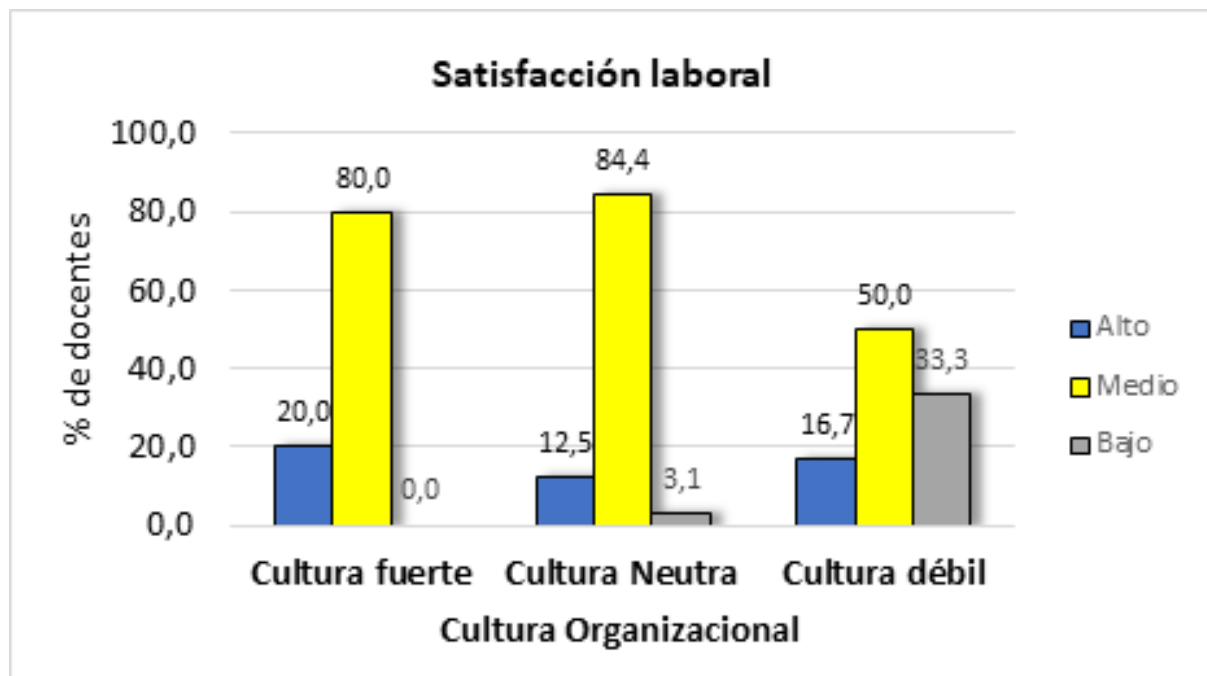

Gráfico 4. Porcentaje de docentes de una universidad de la costa peruana por satisfacción laboral, según cultura organizacional.

\section{Discusión}

Los resultados demuestran que la cultura organizacional influye en la satisfacción laboral de los docentes de la Facultad de Ciencias de la Salud de la Universidad Nacional del Callao. Estos resultados concuerdan con Durand (2015) demuestra que existe una relación significativa entre cultura organizacional y satisfacción laboral, además refiere que existe una moderada relación positiva. Por otra parte, Ibidunni y Agboola (2014), refieren que existe una cultura "correcta" o "mejor" para todas las organizaciones. La cultura más apropiada para una organización es la que mejor le ayuda a hacer frente a las exigencias de su entorno empresarial. Asimismo, Yuang, Jia y Zhao (2016), encontraron que la identificación organizacional moderaba la asociación entre la cultura organizacional y la satisfacción laboral. Encontrando puntajes más altos en satisfacción laboral que aquellos con baja cultura organizacional. Con respecto a la cultura organizacional de los docentes, el $66.7 \%$ cuentan con una cultura organizacional neutra, el 20.8\% cuentan con una cultura organizacional fuerte y el $12.5 \%$ cuentan con una cultura organizacional débil. 
Con respecto a la variable satisfacción laboral, Alfaro, Leyton, Meza y Sáenz (2012), encontraron que los trabajadores tienen un nivel de satisfacción laboral medio; y que existen diferencias significativas por condición laboral respecto al nivel de satisfacción en sus diversos factores en cada una de ellas. Datos que concuerdan con el presente estudio donde se encontró que el 79.2\% indicaron tener una satisfacción laboral media, seguido del $14.6 \%$ de docentes que presentaron una satisfacción laboral alta y solo el $6.3 \%$ de docentes presentaron una satisfacción laboral baja.

Por otra parte, Muñoz (2019), encontró una correlación significativa y positiva entre las dimensiones de la variable cultura organizacional y los factores de satisfacción laboral. Cotocel, Lovan, Gogănău, Nechita, Deca, Cotoi y otros (2019) en los resultados de su estudio demostraron que los docentes se encuentran satisfechos con la organización donde se respeta y reconoce los méritos profesionales, asimismo la satisfacción laboral ya que se respeta las oportunidades de desarrollo, Cheung y Lun (2015), examinaron la asociación entre la satisfacción laboral y cultura organizacional. La correlación bivariada mostró que tanto la actuación profunda como la expresión de emociones sentidas naturalmente estaban relacionadas positivamente con las dos dimensiones de satisfacción laboral.

$\mathrm{Al}$ respecto, en el presente estudio se encontró que los docentes que presentaron una cultura organizacional fuerte, el $80 \%$ presentó un nivel de satisfacción laboral medio, el $20 \%$ presentó un nivel de satisfacción laboral alto y ninguno presentó un nivel de satisfacción laboral bajo, del total de docentes que presentó una cultura organizacional neutra el $84.4 \%$ presentó un nivel de satisfacción laboral medio, el $12.5 \%$ presentó un nivel de satisfacción laboral alto y solo el $3.1 \%$ presentó un nivel de satisfacción laboral bajo, del total de docentes que presentó una cultura organizacional débil, el 50.0\% presentó un nivel de satisfacción laboral medio, el 33.3\% presentó un nivel de satisfacción laboral .

\section{CONCLUSIONES}

La relación entre la cultura organizacional y la satisfacción laboral de los docentes del Facultad de Ciencias de la Salud de la Universidad Nacional del Callao, evidencian que existe una regular fuerza de relación entre dichas variables y que si la cultura organizacional se mejorará así también lo hará la satisfacción laboral.

\section{REFERENCIAS}

Alfaro, R., Leyton S., Meza, A. y Sáenz, I. (2012). Satisfacción laboral y su relación con algunas variables ocupacionales en tres municipalidades. Disponible en: http://tesis.pucp.edu.pe/ repositorio/bitstream/handle/

Cheung, F. y Lun, V. (2015). Satisfacción laboral y cultura organizacional: una investigación en educadores de China. J Gen Psychol 142 (4), 253-72. DOI: 10.1080 / 00221309.2015.1091764

Cotocel, C., Iovan, R., Gogănău, A., Nechita, F., Deca, S., Cotoi, B. y otros. (2019). Cultura organizacional e integración personal en la educación médica: estudio de caso Universidad de Medicina y Farmacia de Craiova, Rumania. Rom J Morphol Embryol, 60 (3), 1053-1058. Disponible en: https://www.ncbi.nlm.nih.gov/ pubmed/31912123

Durand, L. (2015). Cultura organizacional y satisfacción laboral del personal administrativo del Hospital Regional Huacho Disponible: http://repositorio.ucv.edu.pe/ bitstream/handle/UCV/6434/Lino_DLM. pdf? sequence $=1$ \&isAllowed $=y$ 
Ibidunni, O. y Agboola, G. (2014). Prediciendo el desempeño a través de los elementos de la cultura organizacional de seis universidades de Nigeria. Archives of Business Research, 2(6). DOI:10.14738/abr.26.736

Muñoz, V. (2019). Cultura organizacional y satisfacción laboral en los colaboradores de una empresa de servicios de entretenimiento. Disponible en: http://repositorio.urp.edu.pe/ handle/URP/2316

Nosnik, A. (2005). Culturas organizacionales: origen, consolidación y desarrollo. México

Olmos, M. y Socha, K. (2006). Diseño y validación mdeidate jueces expertos del instrumento para evaluar cultura organizacional. Universidad de la Sabana. Disponible en: https://core.ac.uk/ download/pdf/47066084.pdf

Palma, S. (2005) Escala de satisfacción laboral (SLSPC). Lima: Cartolan E.I.R.L
Pedraza, A., Obispo, S., Vásquez, G. y Gómez, G. (2015). Cultura organizacional desde la teoría de Edgar Schein: Estudio fenomenológico. Clío América, 9 (17),17-25. file:///C:/Users/ LENOVO/Downloads/Dialnet-

Rodríguez, D., De León, L. y Galarza, J. (2015). Los retos actuales de las instituciones de educación superior en el área de la gestión. Revista Universidad y Sociedad, 7(3), 52-55. Disponible en: http://rus.ucf.edu.cu/

Yuan, G., Jia, L. y Zhao, J. (2016). Organizational Identification Moderates the Impact of Organizational Justice on Job Satisfaction.0 (0), 189-195. DOI: 10.3233/WOR-162271 